from education than women. Conclusions: Cognitive reserve is an important mediator on the pathway between social isolation and cognitive function, partially buffering the negative effect that social isolation has on cognitive function. Older individuals, particularly socially isolated ones, may benefit from maintaining a cognitively active lifestyle.

\section{O4-08-03 PSYCHOSOCIAL OUTCOMES OF APOE E4 GENOTYPE DISCLOSURE IN THE GENERATION STUDY}

Angela R. Bradbury ${ }^{1}$, Jason Karlawish ${ }^{1}$, J Scott Roberts ${ }^{2}$, Brian L. Egleston ${ }^{3}$, Linda Patrick-Miller ${ }^{4}$, Elisabeth McCarty Wood $^{1}$, Scott YH. Kim ${ }^{5}$, Jan Jaeger ${ }^{1}$, Neeraja Reddy ${ }^{1}$, Cara Cacioppo $^{1}$, Dare Henry-Moss ${ }^{1}$, Stephanie Jideama ${ }^{1}$, Demetrios Ofidis ${ }^{1}$, Fonda Liu ${ }^{6}$, Angelika Caputo ${ }^{7}$, Marie-Emmanuelle Riviere ${ }^{8}$, Mauritz Bezuidenhoudt ${ }^{8}$, Carolyn Langlois ${ }^{9}$, Eric M. Reiman ${ }^{10}$, Pierre N. Tariot ${ }^{9}$, Jessica B. Langbaum ${ }^{10},{ }^{1}$ University of Pennsylvania, Philadelphia, PA, USA; ${ }^{2}$ University of Michigan, Ann Arbor, MI, USA; ${ }^{3}$ Fox Chase Cancer Center, Philadelphia, PA, USA; ${ }^{4}$ Independent Consultant, Chicago, IL, USA; ${ }^{5}$ National Institutes of Health, Bethesda, MD, USA; ${ }^{6}$ Novartis Pharmaceuticals Corporation, East Hanover, NJ, USA; ${ }^{7}$ Novartis Pharma AG, Basel, Switzerland; ${ }^{8}$ Novartis Pharma, Basel, Switzerland; ${ }^{9}$ Banner Alzheimer's Institute, Phoenix, AZ, USA $;{ }^{10}$ Arizona Alzheimer's Consortium, Phoenix, AZ, USA. Contact e-mail: angela.bradbury@uphs.upenn.edu

Background: The Generation Study 1 is an Alzheimer's prevention trial recruiting 60-75 year old APOE $e 4$ homozygotes. Participants have to learn their APOE $e 4$ genotype to screen for this trial. While some studies have reported favorable psychosocial outcomes after APOE genotype disclosure, outcomes in homozygotes and diverse populations remain limited. Methods: Participants are recruited through the Alzheimer's Prevention Initiative Generation Program, enrolling APOE $e 4$ carriers for preclinical Alzheimer's disease trials. A pre-disclosure educational video informs participants about Alzheimer's disease, APOE and considerations for learning their APOE results. Genotype disclosure is provided remotely (phone or videoconference) with a genetic counselor or on-site with a genetic counselor or physician with expertise in neurogenetics. Participants complete pre-disclosure, post-disclosure (2-7 days), 6 week and 6 month measures including genetic knowledge, result recall, perceived risk, state anxiety, depression, disease-specific anxiety, positive and negative responses, uncertainty, satisfaction and health behaviors. T-tests and ANOVAs were used to compare groups. Results: As of Oct 2017, 151 participants have received APOE $e 4$ results, including 61 homozygotes, 46 heterozygotes and 44 noncarriers. The mean age of participants is 65.9 (SD 4.21), $76 \%$ are female. Homozygotes had less decline in state anxiety than non-carriers and heterozygotes at post-disclosure (homozygotes 0.0 , SD 3.01, non-carriers 0.0, SD 3.83, heterozygotes -1.7, SD 3.33). Yet, subgroups only showed small differences in change in state anxiety at 6 weeks or 6 months. Homozygotes had increases in disease-specific distress post-disclosure, while the other groups had declines (homozygotes +2.3, SD 6.70, non-carriers -1.1, SD 7.21, heterozygotes -0.4 , SD 5.48). At 6 weeks and 6 months, genotype subgroups had only small differences in change in disease-specific distress. There were only small differences between groups in change in depression at any time point. Conclusions: Initial results suggest that while APOE $e 4$ homozygotes experience less decline in anxiety and distress after result disclosure, both decline over time and don't differ from heterozygotes and non-carriers at 6 months. Ongoing data collection will provide an opportunity to evaluate additional participant outcomes and factors associated with more favorable outcomes.

\section{O4-08-04 A COMPREHENSIVE MODEL OF FACTORS ASSOCIATED WITH SUBJECTIVE PERCEPTIONS OF LIVING WELL WITH DEMENTIA: FINDINGS FROM THE IDEAL STUDY}

Linda Clare ${ }^{1}$, Yu-Tzu Wu ${ }^{1}$, Ian Rees Jones ${ }^{2}$, Christina R. Victor ${ }^{3}$, Sharon M. Nelis ${ }^{1}$, Anthony Martyr ${ }^{1}$, Catherine Quinn ${ }^{1}$,

Rachael Litherland ${ }^{4}$, James Pickett ${ }^{5}$, John Vincent Hindle ${ }^{6}$, Roy W. Jones ${ }^{7}$, Martin Richard John Knapp ${ }^{8}$, Michael Kopelman ${ }^{9}$, Robin G. Morris ${ }^{10}$, Jennifer M. Rusted ${ }^{11}$, Jeanette M. Thom ${ }^{12}$, Ruth A. Lamont ${ }^{1}$, Catherine Henderson $^{13}$, Isla Rippon ${ }^{3}$, Alexandra Hillman ${ }^{2}$, Fiona Matthews ${ }^{14}$, the IDEAL Study Team, ${ }^{1}$ University of Exeter, Exeter, United Kingdom; ${ }^{2}$ Cardiff University, Cardiff, United Kingdom; ${ }^{3}$ Brunel University, Uxbridge, United Kingdom; ${ }^{4}$ Innovations in Dementia Community Interest Company, Exeter, United Kingdom; ${ }^{5}$ Alzheimer's Society, London, United Kingdom; ${ }^{6}$ Bangor University, Bangor, United Kingdom; ${ }^{7}$ Research Institute for the Care of Older People, Bath, United Kingdom; ${ }^{8}$ London School of Economics and Political Science, London, United Kingdom; ${ }^{9}$ King's College London, London, United Kingdom; ${ }^{10}$ King's College London, Institute of Psychiatry, London, United Kingdom; ${ }^{11}$ University of Sussex, Falmer, United Kingdom; ${ }^{12}$ University of New South Wales, Sydney, Australia; ${ }^{13}$ London School of Economics, London, United Kingdom; ${ }^{14}$ Newcastle University, Newcastle upon Tyne, United Kingdom. Contact e-mail: l.clare@exeter.ac.uk

Background: The concept of 'living well' is increasingly used to indicate that it is, or should be, possible for a person living with dementia to experience a subjective sense of 'comfort, function and contentment with life.' We used a theoretically-derived conceptual framework to investigate capability to 'live well' with dementia through identifying the relative contribution of domains associated with the subjective experience of living well. Methods: We analysed data from 1550 community-dwelling individuals with mild to moderate dementia participating in the baseline wave of the Improving the experience of Dementia and Enhancing Active Life (IDEAL) cohort study. Subjective perceptions of ability to live well were obtained by generating a living well latent factor from responses on the Quality of Life in Alzheimer's disease (QoL-AD), Satisfaction with Life and WHO-5 Well-being scales. Multivariate modelling and structural equation modelling was used to investigate variables potentially associated with living well. Variables were grouped into five domains, latent variables were constructed representing Social Location, Capitals, Assets and Resources, Psychological Characteristics and Psychological Health, Physical Fitness and Health, and Managing Everyday Life with Dementia, and associations with living well were examined. All models were adjusted for age, sex and dementia sub-type. Results: Considering the domains singly, the Psychological Characteristics and Psychological Health domain was most strongly associated with living well (3.56; 95\% CI: 2.25 , 4.88), followed by Physical Fitness and Physical Health $(1.10,95 \%$ CI: -2.26, 4.47). Effect sizes were smaller for Capitals, Assets and Resources (0.53; 95\% CI: -0.66, 1.73), Managing Everyday Life with Dementia $(0.34 ; 95 \%$ CI: $0.20,0.87)$, and Social Location (-0.12; 95\% CI: $-5.72,5.47)$. Following adjustment for the Psychological Characteristics and Psychological Health domain, other domains did not show independent associations with living well. Conclusions: Psychological resources are central to subjective perceptions of living well and offer important targets for immediate intervention. Availability of social and environmental resources, and physical fitness, underpin these positive psychological states, 